\title{
Biomaterials Targeting AMD: Are we there yet?
}

Rachel Lawless (nee McCormick) ${ }^{1}$, Veronica Hidalgo-Alvarez ${ }^{1}$, and Atikah Haneef ${ }^{1^{*}}$

${ }^{1}$ Institute of Life Course and Medical Sciences, Department of Eye and Vision Science, The University of Liverpool, Liverpool, United Kingdom

*Correspondence: Dr Atikah Haneef, atikahh@liverpool.ac.uk

Abstract: Age-related macular degeneration (AMD) is the leading cause of central blindness in developed countries. It affects people mainly over the age of 50 years. It is a disease of the macula, an area of the retina responsible for sharp central vision. It particularly affects the Bruch's membrane (BM); a layer in the retina that acts as the basement upon which retinal pigment epithelial cells (RPE) attach and survive. The pathology of AMD is not fully understood, but age is considered the main risk factor. There are two forms; nonexudative, leading to the end-stage of the disease, called nonexudative (or dry) AMD ( $90 \%$ of cases) where fatty deposits called drusen form under the RPE on top of the BM lifting off the RPE, and neovascular (or wet) AMD ( $10 \%$ of cases) where abnormal new blood vessels grow and push through the BM, bleeding in and disrupting the RPE. Neovascular AMD is well controlled with regular antiangiogenic drug injections of anti-vascular endothelial growth factor (anti-VEGF) into the eye, whereas there is no current treatment for nonexudative AMD. Many research groups across the world are working on a treatment for nonexudative AMD. This review discusses the research currently being conducted including cell therapies, development of cell transplantation membranes, targeting other disease structures in affected retina (i.e. drusen), and drug delivery to the retina using nanoparticles. Finally, we include our research contributing to the field; developing a bioactive membrane intended to function twofold: target diseased structures and transplant healthy RPE to the desired area.

Keywords: AMD, Biomaterials, electrospinning, membranes, Bruch's membrane

\section{AMD: The Background}

Age-related macular degeneration (AMD) is a debilitating blindness predominantly affecting people over the age of 50 years. Pathology of the disease is yet unknown, but there are multiple genetic and environmental factors that contribute to risk of developing AMD[1]. These include; smoking[2], oxidative stress caused by smoking[3], and poor nutritional diet[4]. It is well accepted, however, that AMD is a major consequence of aging[5-8]. There are multiple genetic mutations which have been shown to increase the risk of AMD[9], particularly those involved with the complement system[10, 11].

There are two forms of AMD; neovascular and nonexudative (Figure 1). It is possible to suffer from both forms of AMD simultaneously or to develop neovascular AMD after being diagnosed with the nonexudative form[12]. Neovascular AMD makes up $10 \%$ of all cases and is due to overproduction of vascular endothelial growth factor (VEGF). This causes the development of abnormal blood vessels from the underlying blood supply to the retina, the choroid, to push through the BM and retinal pigment epithelial cells (RPE) $[13,14]$ resulting in haemorrhaging and scar formation[12]. If left untreated, this can cause vision loss in a matter of days, however current clinical treatment has a very high success rate. Regular injections of anti-VEGF into the vitreous humor reverse and prevent formation of any further abnormal blood vessels, therefore preserving patients' sight. 
Nonexudative AMD makes up $90 \%$ of all cases involving a disruption in the transport of waste and nutrients across the retina between the RPE cells and the choroid, due to accumulation of material between the RPE cells and the BM. These accumulations are known as drusen and are mainly comprised of lipids $[15,16]$. Although drusen are considered benign deposits with no active role in causing active damage to the structures in the eye, as they grow in size they can begin to lift the RPE cells off and away from the BM layer[17]. As the RPE are an adherent cell, they require a basement membrane to survive and are highly active, requiring a thorough nutrient/waste exchange system due to the high throughput nature of the retina, these pathological changes to the BM resulting in growth of drusen and blockage of transport result in RPE atrophy and cell death. Consequently, photoreceptor cells on the RPE also undergo cell death causing eventual central vision loss; the end stage of the disease called nonexudative AMD[12]. Once sight is lost, it is lost permanently and cannot be restored. Therefore, regaining RPE function is essential to preventing onset of blindness caused by nonexudative AMD.

Currently there is no treatment or cure for nonexudative AMD, but many research groups across the world are investigating various methods to develop a treatment. There are numerous routes to tackling such a disease; from the molecular level to the macro level. This review provides an overview of the current developments in using biomaterials as RPE transplantation substrates and also on the research that target the reduction/removal of fatty deposits in the retina as potential routes for treating nonexudative AMD. To conclude this review, we have also described how our research is contributing to the continual development of this research area.

\section{Numerous approaches for cell transplantation}

\section{Cell suspensions and cell sheets:}

Transplantation of cells aiming to treat AMD has been widely investigated in humans[18]. Different cell types have been tested in this endeavour including RPE, iris pigment epithelial cells (IPE), retinal progenitor cells (RPC), photoreceptors, and stem cells[19]. Cell suspensions is the simplest route to cell insertion, of which injections into the retina have been extensively researched. A study by Schwartz et al injected 18 patients with a suspension of human embryonic stem cell-derived RPE (hESC-RPE) and reviewed over 4 years. Improved visual acuity was reported with no adverse reactions. Due to the allogeneic nature of the cells, however, patients were given immunosuppressant drugs six week prior to and six weeks post injection, which was criticised due to the induced side-effects that could have been avoided[20].

Similarly, a study found good cell-sheets formed in vitro from a suspension of donor induced pluripotent stem cells (iPSCs)-derived RPE (iPSCE-RPE)[21]. This proceeded to go into clinical trials where a patient with advanced wet AMD was injected with iPSC-RPE cell suspension. This clinical trial was halted due to the patient undergoing retinal oedema after injection of the cell suspension, requiring surgical intervention. Despite the failure of this trial, the iPSCs had been matched with the human leukocyte antigen (HLA) of the patient obviating the need to use immunosuppressant drugs. The team postulate that the reaction occurred due to backflow of fluid the cells were suspended in, rather than a reaction to the cells themselves. Typing HLA can be a limiting factor since it is dependent on the ethnicity of the target population, but this also leaves the potential to allow the use of a limited number of selected donors for iPSC-RPE being generated with a known HLA-type[22, 
23]. This trial also made clear that a very robust safety trial must be undertaken before any approach towards humans is made.

The disappointing outcome of injecting cell suspensions highlights the need to develop an alternative route to cell transplantation; one which does not require a cell suspension fluid, potentially eliminating the reported fluid backflow. Furthermore, transplantation of RPE cells without repairing the structural damage caused in the BM during development of AMD does not lead to the therapeutic improvements sought in clinic[18]. To eliminate these complications, development of cell-sheets to transplant onto the native BM are being undertaken, with an aim that the cell-sheet will eventually secrete their own extracellular matrix (ECM) once reaching confluence[24-26]. This would restore the structural characteristics of the BM, thus supporting growth of the cell-sheet.

Questions arise, however, on how similar the composition of transplanted-cell secreted ECM is to native ECM and how long it would take to generate new ECM due to the limited amount of ECM the RPE is able to secrete[27], suggesting transplanted cell-sheets would not be able to generate a mechanically robust enough basement membrane. Thus, tissue engineering approaches, based on utilisation of natural or synthetic BM substitutes, have been adopted in order to increase efficiency of cell transplantation therapies by ensuring an appropriate cell engraftment into the ECM[19] (Figure 2).

\section{Biomaterials for dry AMD}

Biodegradable matrices have been investigated as a possible middle-ground, providing supportive scaffolds to delicate cell monolayers while secreting their own ECM. Substrates include decellularized lens capsule, BM, Descemet's membrane or amniotic membrane, which have close composition and morphology to native tissue. Limited availability of donors and potential transmission of diseases, however, make it desirable to seek alternative substitutes[25, 28]. Natural polymers such as collagen, fibrin or silk are biocompatible and have similar composition to natural ECM; but potential for inflammatory response and challenging mechanical properties have led to interest in synthetic biomaterials $[25,29]$.

Cell-support membranes paved the way for development of synthetic BM as an option for cell delivery, with much research investigating both degradable and non-degradable materials. Due to the plethora of materials being investigated, we have concentrated on the materials that are undergoing pre-clinical or clinical trials.

Da Cruz et al investigated commercially available poly(ethylene terephthalate) PET transwell inserts, inserting them into two patients, over 70 years, who had lost their vision in one eye (unable to read) [30]. These inserts are non-biodegradable, porous and coated with human vitronectin to grow hESCs to maturity. Researchers ensured stem cells had differentiated into RPE cells (hESC-RPE) by staining with relevant characteristic proteins before insertion. Patients were monitored over 12 months; one patient had their sight restored, nonetheless their sample group is very small, which has been highlighted as a setback[23].

Parylene-c is another synthetic non-degradable polymer, much like the aforementioned commercially available PET transwells[31]. Unlike transwells, parylene-c is fabricated using soft 
lithography into regularly patterned substrates with ultra-thin submicron scale sections, mounted on a mesh frame to provide mechanical strength. Submicron regions are permeable to water and water-soluble ions that makes it optimal for diffusion systems[32]. Initial results from this trial show hESC-RPE seeded onto parylene-C membranes were well-tolerated when subretinally inserted into 5 patients with advanced AMD. Subjects have reported improvement in vision in treated eyes, compared to non-treated eyes, attributed to rehabilitation of dormant photoreceptors in the area by their implant. They suggest cell-seeded hESC-RPE promoted formation of outer segments in the area of geographic atrophy thereby contributing to improvement of vision[32]. Parylene-c has been undergoing active clinical trials since October 2015 which is due to end in June 2023[33].

\section{Materials to mimic native BM topography}

Although the above-mentioned substrates have reached clinical trials these substrates do not match nor mimic native ECM of the BM: a randomly orientated fibrous meshwork with an open network of voids. A number of groups have recognised this limitation and are using electrospinning to try to mimic more closely the native BM.

A range of biodegradable polymers have been investigated and discussed at length; including gelatine/polycaprolactone, poly(lactic-co-glycolic acid) (PLGA) and silk fibroin with fibre diameters of 150-400nm[34]. Poly(L-lactic acid) (PLLA) fibres with a diameter of 70nm have been reported, which when coated with laminin allowed RPE cells to grow with phenotypic morphology exhibiting typical apical microvilli as well as exhibiting phagocytic activity[35]. Pre-clinical trials on rats showed PLLA mesh was well tolerated in the retina and could be a potential candidate for a synthetic BM. Despite the potential of these scaffolds, having a biodegradable substrate as a basement membrane for RPE leaves open a range of questions: what happens when the substrate has fully degraded? Will the cells have enough time to secrete their own ECM? Will the cells be left in a "diseased state" after the substrate has been fully absorbed? Considering RPE require a stable basement membrane, these are relevant questions.

To address this, fabricating synthetic fibrous membranes using non-degradable polymers has been investigated. Electrospinning of non-degradable polymers such as PET on commercially available PET transwells has been shown to exhibit good tolerance following subretinal acellular insertion in rabbits, with good RPE cell morphology cultured in vitro on nanofibrous structures (200nm fibre diameter) compared to planar counterparts[36]. These nanofibrous PET scaffolds lacked mechanical strength and had to be mounted on to commercial transwells, which have been described as having limited porosity[37]. They also showed cell invasion into their composite membrane, thus lacking the properties of the native BM-RPE. Therefore, there is scope to fabricate a nanofibrous substrate that mimics topography of the native BM, as well as possesses mechanical integrity. Our work addresses this and we will go into further detail as to the contribution of our research at the end of this review.

\section{Other targets in AMD treatment}

\section{$\underline{\text { Targeting drusen in } A M D}$}


As discussed above, formation of sub-RPE drusen contribute to loss of BM permeability, leading to death of RPE cells and subsequently photoreceptor cells. Drusen thus pose a major risk factor for AMD progression[38].

Although much research has focused on inserting new cells to replenish dying RPE cells, the underlying problem of a diseased BM remains. Consequently, research has also strived to tackle AMD by using biological agents to breakdown drusen with aims of unblocking the BM and restoring transport between the choroid-RPE barrier, preventing further loss of photoreceptors. There is supportive clinical evidence for removal of soft drusen to treat both neovascular and exudative $A M D[39]$. Research has shown that higher content of hydrophobic esterified cholesterol within BM increases resistance to fluid transport in the BM; while removing the internal layers of elastin in the BM decreases resistance to fluid transport[40]. Suggesting removal of fatty deposits could be beneficial to restoring fluid movement across the choroid-RPE barrier.

Drusen are generally split into two morphological phenotypes; hard and soft. Hard drusen are yellow, small $(<63 \mu \mathrm{m})$, punctate, and generally not seen as a direct risk for AMD progression[41] . Hard drusen appear in all areas of the retina, but macular drusen and peripheral drusen differ in composition. Peripheral hard drusen are smaller, more homogenous, contain more shells, and are more frequent in the periphery than macular drusen[41]. Multiple hard drusen in the macular increase risk of soft drusen and RPE aberrations presenting, which increase likelihood of nonexudative and neovascular AMD developing.

Soft drusen are filled with membranous debris[38] are dome shaped with sloped sides[42], and are found only in the macular[41]. There is supportive clinical evidence for removal of soft drusen to treat both neovascular and nonexudative AMD[39].

Drusen have been compared to atherosclerotic plaque, due to both containing similar protein molecular composition and exhibiting lipoprotein retention[43]; therefore, some groups have taken to treating $\mathrm{AMD}$ as an atherosclerosis-like disease. Rudolf et al looked at lipid deposition on the BM in AMD and found that 6 intravitreal injections of anti-inflammatory, anti-atherogenic peptide L-4F over 6 months, resulted in decrease in thickness of $\mathrm{BM}$, restoration of ultrastructure, and reduction in amount of lipids on the BM in Macaca fascicularis. Interestingly, results were seen bilaterally in non-injected control-eyes compared to placebo subjects[44]. The same group also showed that 1 injection of antiatherogenic peptide L-4F was able to remove lipid deposition and restore the BM ultrastructure in mice lacking apoplipoprotein-E, a protein involved in metabolism of fat. Despite successfully removing BM lipids, neither of these studies established whether improvements were made in eyesight of subjects.

Vavvas et al tested the ability of atorvastatin, a hydrophobic statin and well-known treatment for atherosclerosis, to remove drusen in human AMD patients. The original study involved one 63-yearold male, who was given increasing doses of atorvastatin, up to $80 \mathrm{mg} /$ day for six months. Following this he exhibited no evidence of drusen nor RPE atrophy, and also showed improvement in visual acuity by gaining 12 letters, using the Early Treatment Diabetic Retinopathy Study (ETDRS) visual acuity chart. Consequently, a larger human trial was undertaken with 23 patients treated with $80 \mathrm{mg} /$ day of atorvastatin over the course of a year. Nearly half of the patients responded positively towards treatment with an average visual acuity improvement by gaining 3.3 letters, with 8 out of 23 
patients presenting near complete regression of drusen[45]. Therefore L-4F and atorvastatin could potentially be a route towards treatment of nonexudative AMD.

\section{Conflicting data}

Despite evidence for the role of lipid accumulation as a risk factor for developing AMD, conflicting data exists on whether reducing their size would be beneficial in preventing AMD progression[4648]. A meta-analysis of three population-based cohorts over 20 -years failed to show any association between lipid levels, lipid pathway genes or statin use with incidence or progression of AMD[47]. Hence, other drugs have also been tested to target drusen as well as targeting different aspects of AMD. Garcia Filho et al used eculizumab which targets component 5 of the complement system, part of the immune system activated by pathogens to induce an inflammatory response. Eculizumab was given intravenously over a period of 26 weeks with the primary aim to identify $>50 \%$ reduction in drusen size. Eculizumab did not significantly reduce drusen volume and growth of drusen was dependent on the number of complement at-risk alleles[49].

Evidence of the benefits of removing drusen in AMD is conflicting; there are a variety of responses to the drugs in both treated and placebo patients, even in studies which selected a sub-type of drusen. For example, one eye in the placebo group in the above study by Garcia Filho et al exhbited decrease in drusen volume of at least $50 \%$ and two eyes developed neovascularization drusen[45]. Research by Virgili et al found reducing drusen size by laser treatment failed to prevent incidence of neovascularization, geographic atrophy and loss of visual acuity[50]. Furthermore, reduction in drusen size or number may not necessarily be beneficial and may be an indication of AMD progression to geographic atrophy or macular neovascularisation; providing evidence that removing or reducing size of drusen is not enough to treat and prevent AMD[51].

This therefore suggests although drusen are a compounding indicator in progression of AMD, simply removing them is not the answer; a successful treatment would be one that addresses the underlying problems within both the diseased BM and RPE cells. Moreover, there are multiple facets to address in order to achieve targeted delivery of drugs to the retina.

\section{Delivery of drugs}

The short-term delivery methods used in the aforementioned studies required frequent direct ocular injections or systemic dosages. If translated to clinic, frequency and delivery method would lead to a time-consuming and uncomfortable experience for patients. One existing example is the multiple anti-VEGF injections patients with neovascular AMD have to be administered directly into the vitreous every month. Evidence has shown multiple injections increase risk of cataract formation, endophthalmitis, retinal detachment and vitreous haemorrhage[52,53]. Physical trauma of injections induces an inflammatory response leading to long-term eye damage, suggesting frequency of injections should be kept to a minimum. Additionally, due to the nature of this method, patients are less likely to be compliant with appointments and treatment[54], supporting the need for an improved sustained drug-delivery system. These issues could be overcome by developing drug delivery systems for the administration of anti-VEGF treatments or any other drugs that could be used to treat AMD[55]. Thus, numerous studies are being conducted in order to create novel formulations to provide controlled release of therapeutic agents that target this disease. Some examples of these systems include hydrogels, liposomes, microparticles, nanoparticles and 
microneedles [55-58]. In order to increase targeting efficiency of these formulations and achieve a higher degree of control, stimuli-responsive drug delivery systems have been created. These systems have the ability to trigger release of therapeutic agents only when subjected to a stimulus that is present exclusively in the diseased tissue, such as variations in $\mathrm{pH}$ and temperature or presence of specific enzymes[59, 60].

A well-established delivery system is the silicone oil (SIO) tamponade which has been used clinically since 1958, usually for eye surgeries such as proliferative vitreoretinopathy (PVR) or retinal detachment/tears. Due to the close proximity to the retina, SIO can be dosed with drugs[61]. Despite its historical use, it is not without its drawbacks; a study showed $17.5 \%$ of neovascular AMD patients who underwent macular translocation surgery, followed by a SIO tamponade, went on to develop retinal detachment one month after removal[62] highlighting a need for an improved drug delivery system to the retina.

\section{Nanoparticle delivery to retina}

Delivery of drugs to the retina is particularly difficult due to its anatomical positioning and highly restrictive blood-retinal barrier that regulates much of the ion, protein, and water movement across it[63]. It is essential for drug delivery to be as effective as possible; therefore, nanoparticles have been investigated as a method for sustained, localised drug delivery.

Nanoparticles (NPs) have been shown to protect biological activity of drugs, exhibit their sustained controlled release, prevent degradation of encapsulated proteins and cross the blood-retinal barrier $[64,65]$. Moreover, they can also be used as non-viral gene delivery vectors, reducing risks associated with using viral vectors, which include development of immune responses and reversion of the virus to its wild-type strain. As AMD affects RPE and photoreceptor cells, NPs can be used to deliver a healthy copy of the mutated gene to these cells[66]. Previous studies performed in mice showed that NPs were successful in delivering a gene encoding green fluorescent protein (GFP) into the subretinal space, resulting in expression of this protein in both photoreceptors and RPE cells. GFP expression could be detected as early as $2-4$ days after injection and was maintained for several weeks $[67,68]$.

Bourges et al successfully delivered PLA NPs containing fluorescent dye through intravitreal injections into rat retina. There was no toxic effect; only a mild inflammatory response which had decreased markedly by 48 hours. Staining in retina and RPE cells confirmed dyes were being released with effects being seen 4 months after injection[68]. Similarly, Patel et al used lipid NPs to successfully deliver messenger RNA to the retina, evidenced by gene expression that lasted up to 5 days[69]. Zhang et al encapsulated dexamethasone, a routinely utilised drug to treat ocular diseases, in PLGA NPs and intravitreally injected them into rabbit retina. No side effects were apparent and NPs sustainably released dexamethasone at a concentration of $3.85 \mathrm{mg} / \mathrm{L}$ over 30 days[70]. Varshochian et al also reported bevacizumab encapsulated albuminated-PLGA NPs were able sustainably release the drug at a concentration of $500 \mathrm{ng} / \mathrm{mL}$; the known concentration for angiogenesis inhibition[71].

Additionally, there have been reports of drug-loaded NPs successfully being applied for their intended function: Xu et al inhibited laser induced choroidal neovascularisation (CNV) via intravitreally injected dexamethasone acetate-loaded PLGA NPs in rats[72] and Tabatabaei et al 
inhibited retinoblastoma cell viability in vitro using lipid NPs encapsulated with microRNA and melphalan, a chemotherapeutic agent[73]. A recent study conducted by Narvekar et al showed that PLGA NPs could be used to sustainably release Axitinib which inhibited VEGF-angiogenesis in ARPE19 cells[74]. Furthermore, drug-loaded NPs have entered clinical trials; paclitaxel albumin-stabilized NPs to treat metastatic melanoma of the eye[75] and dexamethasone-cyclodextrin NPs for treating diabetic macular oedema[76]. This represents the positive therapeutic potential drug-encapsulated NPs have on ocular diseases. Moreover, NPs have the potential to be fabricated in a variety of internal morphologies, which potentially could allow the delivery of multiple drugs/compounds/factors through one dose[77-79] (Figure 3). Another novel approach for the treatment of wet AMD is photodynamic therapy (PDT), based on delivery of a photosensitiser to the CNV. In order to prevent delivery of photosensitiser to healthy retinal or choroidal blood vessels, nanotechnology-based PDT has been tested in animal models. To achieve this, a dendrimer porphyrin was encapsulated in a polymeric micelle, which led to a high accumulation of the photosensitiser in the abnormal vessels and a subsequent increase in efficacy of the treatment[80].

\section{Conclusion \& Future Direction}

From the vast amount of research in this field, it is apparent that much progress has been made in the search to find a treatment for AMD. However, there are two distinct routes that are chosen to address a treatment for nonexudative AMD; biomaterials to transplant healthy cells to the retina, or drugs to target drusen/fatty deposits residing in the retinal layers.

Our research group is aiming to combine these two routes to develop a composite implant; a persistent biologically active cell transplant membrane. The technology is anticipated to function two-fold: to transplant healthy cells to the desired site while targeting drusen for their removal.

We anticipate fabricating a permanent biocompatible electrospun mesh, a persistent synthetic basement membrane where new RPE cells will be cultured on the apical side. This membrane will be an open network of voids that is made up of randomly orientated non-degradable fibres, allowing transplantation of a monolayer of healthy RPE cells to the required area. Additionally, we envisage decorating the underside side of the membrane with biodegradable NPs. They will encapsulate enzymes/drugs/other lipid-targeting biological factors that will break down the fatty deposits/drusen (Figure 4).

The persistent substrate has already been designed[81, 82] and work has been undertaken to optimise fabrication of nanoparticles and encapsulation of detectable compounds to measure nanoparticle degradation and sustained release[83].

Our work contributes positively towards developing a potential treatment for nonexudative AMD. It targets both the issue of the underlying diseased structures as aforementioned and the need to transplant new RPE cells to the macula. Our work is the first to focus on developing a composite persistent, biologically active cell implant to address nonexudative AMD. 


\section{Figures}

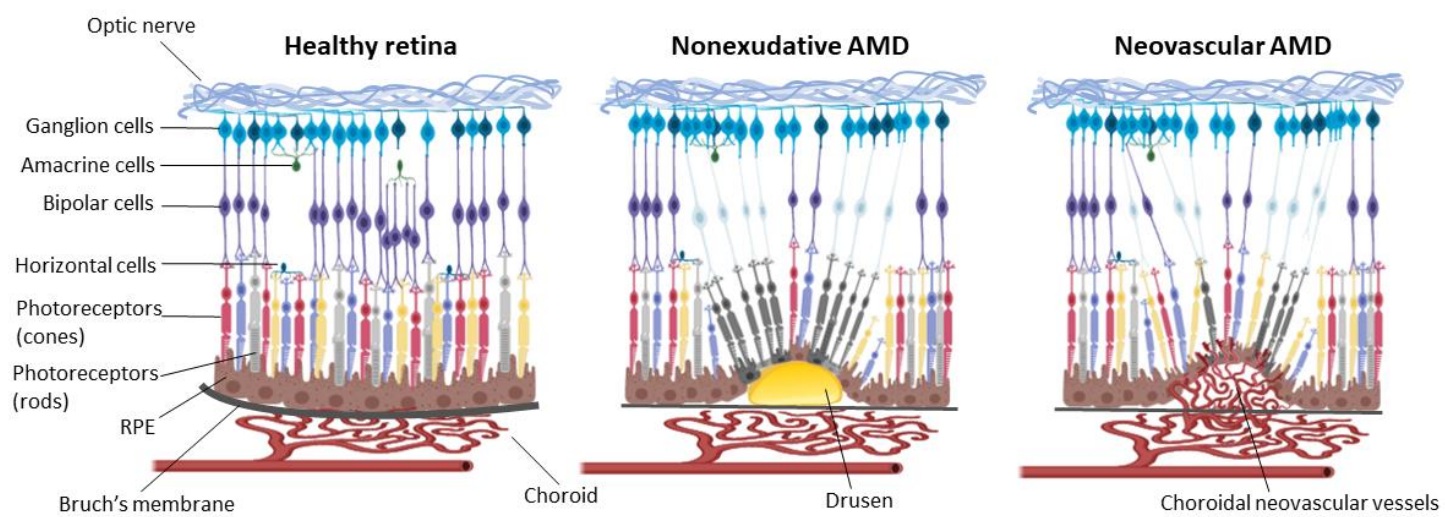

Figure 1: Depicting structures of a healthy retina (left); retina with nonexudative AMD; and retina with neovascular AMD (right) and the resulting disruption of cells.

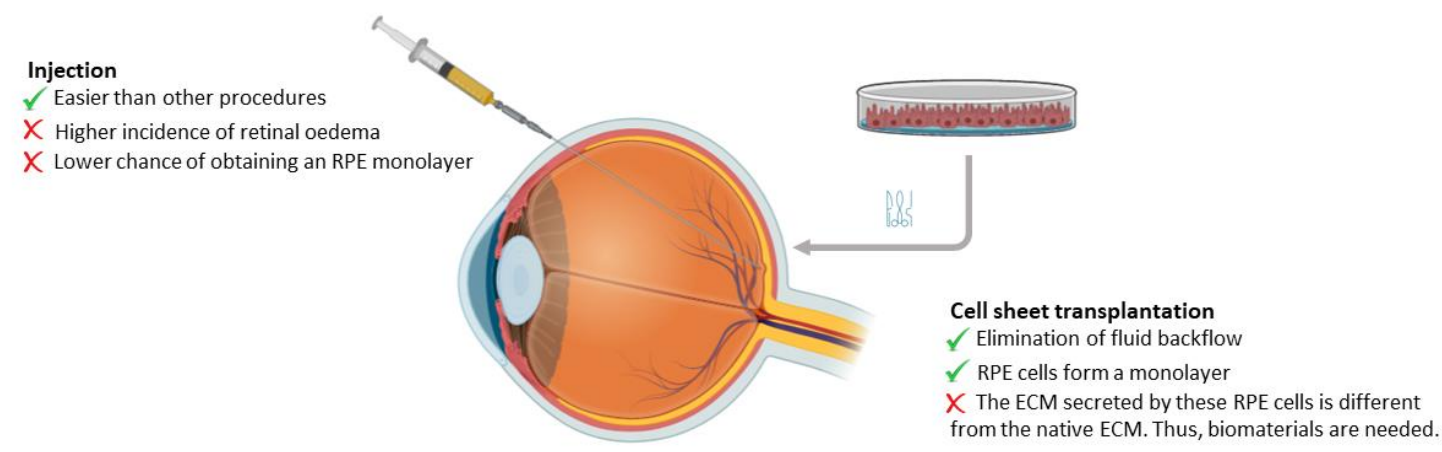

Figure 2: Diagram of two common cell therapy approaches; injection and cell sheet transplantation. While injection is a relatively easy procedure, patients have been reported to suffer from retinal oedema as a consequence of injection. Cell sheet transplantation, therefore, is a promising alternative. However, ECM secreted by transplanted cells have been reported to be different from the native ECM, igniting interest in using biomaterials as a support for transplanted cells.

a

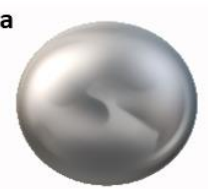

e

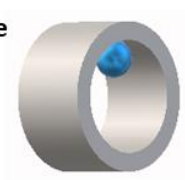

b
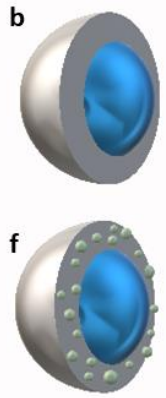

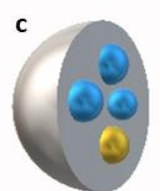

g

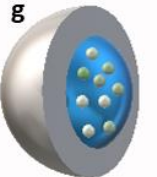

d

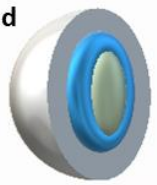

h

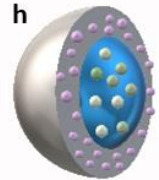

Figure 3: Representation of the various NP internal morphologies possible; single-chamber (a), dualchamber (b), multiple-cores within a shall (c), multiple shells (d), movable core within hollow shell (e), core-shell with smaller particles embedded in shell (f), core-shell with smaller particles 
embedded in core (g), core-shell with smaller particles embedded in both shell and core (h). Adapted from Chaudhuri and Paria 2011, Hayes et al. 2014 and Jegede et al. 2018 [77-79]

a

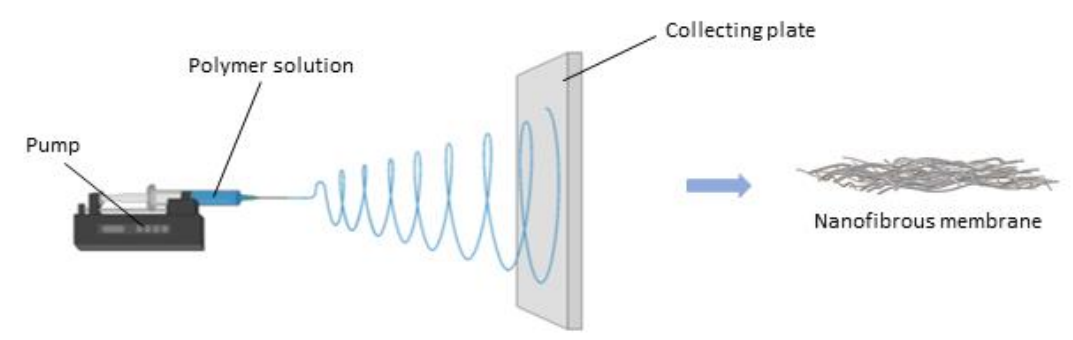

b

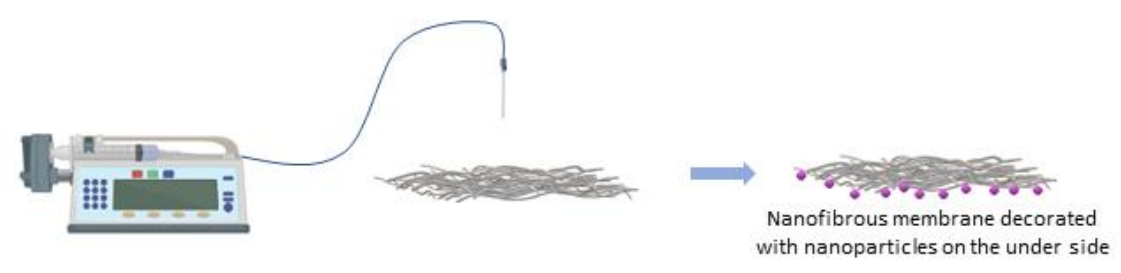

C
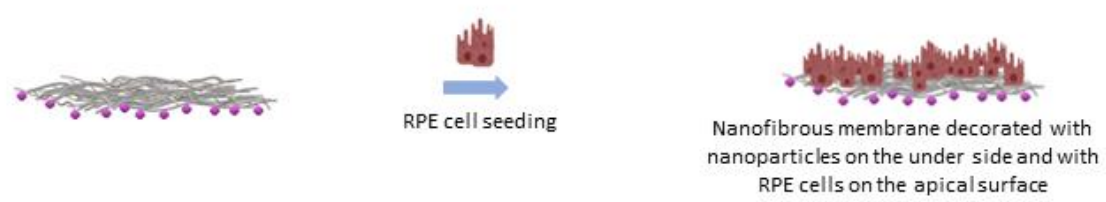

d
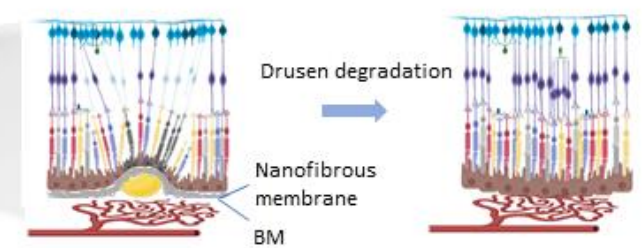

Figure 4: Representation of the step process of our intended work. The persistent nanofibrous membrane is made first using electrospinning (a), followed by electrospraying degradable NPs encapsulated with bioactive substance decorated on the underside of the membrane (b), RPE cells will be cultured on the apical surface of the membrane to form a monolayer of cells (c), finally we anticipate this cultured composite membrane to be surgically inserted subretinally with the intention to target the removal of drusen (d).

\section{Footnotes}

Contributors: RL: planning, literature search, manuscript preparation, manuscript editing, result collection and analyses. VHA: figure preparation. AH: planning, literature search, manuscript preparation, manuscript editing, figure preparation, figure editing, leading research and obtained funding.

Funding: EPSRC, Grant reference number: EP/S001468/1

Competing interests: None.

Copyright information: CC BY. 


\section{References:}

1. Chen, Y., M. Bedell, and K. Zhang, Age-related macular degeneration: genetic and environmental factors of disease. Mol Interv, 2010. 10(5): p. 271-81.

2. Smith, W., et al., Risk factors for age-related macular degeneration: Pooled findings from three continents. Ophthalmology, 2001. 108(4): p. 697-704.

3. Cano, M., et al., Cigarette smoking, oxidative stress, the anti-oxidant response through Nrf2 signaling, and Age-related Macular Degeneration. Vision Res, 2010. 50(7): p. 652-64.

4. Rowan, S., et al., Involvement of a gut-retina axis in protection against dietary glycemiainduced age-related macular degeneration. Proceedings of the National Academy of Sciences, 2017. 114(22): p. E4472-E4481.

5. Risk factors associated with age-related macular degeneration. A case-control study in the age-related eye disease study: Age-Related Eye Disease Study Report Number 3. Ophthalmology, 2000. 107(12): p. 2224-32.

6. Blumenkranz, M.S., et al., Risk factors in age-related maculopathy complicated by choroidal neovascularization. Ophthalmology, 1986. 93(5): p. 552-8.

7. Erke, M.G., et al., Prevalence of age-related macular degeneration in elderly Caucasians: the Tromso Eye Study. Ophthalmology, 2012. 119(9): p. 1737-43.

8. Klein, R., et al., Fifteen-year cumulative incidence of age-related macular degeneration: the Beaver Dam Eye Study. Ophthalmology, 2007. 114(2): p. 253-62.

9. DeAngelis, M.M., et al., Genetics of age-related macular degeneration (AMD). Hum Mol Genet, 2017. 26(R1): p. R45-r50.

10. Maller, J.B., et al., Variation in complement factor 3 is associated with risk of age-related macular degeneration. Nat Genet, 2007. 39(10): p. 1200-1.

11. Seddon, J.M., et al., Rare variants in CFI, C3 and C9 are associated with high risk of advanced age-related macular degeneration. Nat Genet, 2013. 45(11): p. 1366-70.

12. Birch, D.G. and F.Q. Liang, Age-related macular degeneration: a target for nanotechnology derived medicines. Int J Nanomedicine, 2007. 2(1): p. 65-77.

13. Ding, X., M. Patel, and C.C. Chan, Molecular pathology of age-related macular degeneration. Prog Retin Eye Res, 2009. 28(1): p. 1-18.

14. Green, W.R., Histopathology of age-related macular degeneration. Mol Vis, 1999. 5: p. 27.

15. Haimovici, R., et al., The lipid composition of drusen, Bruch's membrane, and sclera by hot stage polarizing light microscopy. Invest Ophthalmol Vis Sci, 2001. 42(7): p. 1592-9.

16. Wang, L., et al., Abundant lipid and protein components of drusen. PLoS One, 2010. 5(4): p. e10329.

17. Mazzitello, K.I., et al. Druse-Induced Morphology Evolution in Retinal Pigment Epithelium. arXiv e-prints, 2016. arXiv:1609.04496.

18. Shadforth, A., et al., A Bruch's membrane substitute fabricated from silk fibroin supports the function of retinal pigment epithelial cells in vitro. Journal of Tissue Engineering and Regenerative Medicine, 2015.

19. Fernández-Robredo, P., et al., Current Treatment Limitations in Age-Related Macular Degeneration and Future Approaches Based on Cell Therapy and Tissue Engineering. Journal of ophthalmology, 2014. 2014: p. 510285.

20. Schwartz, S.D., et al., Subretinal Transplantation of Embryonic Stem Cell-Derived Retinal Pigment Epithelium for the Treatment of Macular Degeneration: An Assessment at 4 Years. Invest Ophthalmol Vis Sci, 2016. 57(5): p. ORSFc1-9.

21. Matsumoto, E., et al., Fabricating retinal pigment epithelial cell sheets derived from human induced pluripotent stem cells in an automated closed culture system for regenerative medicine. PLoS One, 2019. 14(3): p. e0212369.

22. Chichagova, V., et al., Cellular regeneration strategies for macular degeneration: past, present and future. Eye (Lond), 2018. 32(5): p. 946-971. 
23. Ben M'Barek, K. and C. Monville, Cell Therapy for Retinal Dystrophies: From Cell Suspension Formulation to Complex Retinal Tissue Bioengineering. Stem Cells Int, 2019. 2019: $p$. 4568979.

24. Masayuki Yamato, T.O., Cell sheet engineering. Materials today, 2004. 7(5): p. 42-47.

25. Tan, Y.S.E., et al., Tissue engineering of retina and Bruch's membrane: a review of cells, materials and processes. Br J Ophthalmol, 2018. 102(9): p. 1182-1187.

26. Owaki, T., et al., Cell sheet engineering for regenerative medicine: current challenges and strategies. Biotechnol J, 2014. 9(7): p. 904-14.

27. Lu, Y., et al., Recent advances in cell sheet technology for bone and cartilage regeneration: from preparation to application. Int J Oral Sci, 2019. 11(2): p. 17.

28. Hotaling, N., et al., Nanofiber Scaffold-Based Tissue-Engineered Retinal Pigment Epithelium to Treat Degenerative Eye Diseases. Journal of Ocular Pharmacology and Therapeutics, 2016. 32.

29. Ding, S.S.L., et al., Empowering Mesenchymal Stem Cells for Ocular Degenerative Disorders. Int J Mol Sci, 2019. 20(7).

30. da Cruz, L., et al., Phase 1 clinical study of an embryonic stem cell-derived retinal pigment epithelium patch in age-related macular degeneration. Nat Biotechnol, 2018. 36(4): p. 328337.

31. Lu, B., et al., Mesh-supported submicron parylene-C membranes for culturing retinal pigment epithelial cells. Biomed Microdevices, 2012. 14(4): p. 659-67.

32. Ortigoza-Diaz, J., et al., Techniques and Considerations in the Microfabrication of Parylene $C$ Microelectromechanical Systems. Micromachines (Basel), 2018. 9(9).

33. Study of Subretinal Implantation of Human Embryonic Stem Cell-Derived RPE Cells in Advanced Dry AMD. https://ClinicalTrials.gov/show/NCT02590692.

34. E.Y.S. Tan, S.L.S., W.Y. Yeong, Scaffolds for retinal repairs. Handbook of Tissue Engineering Scaffolds: Volume Two, 2019: p. 673-691.

35. Surrao, D.C., et al., Design, development and characterization of synthetic Bruch's membranes. Acta Biomater, 2017. 64: p. 357-376.

36. Liu, Z., et al., Enhancement of retinal pigment epithelial culture characteristics and subretinal space tolerance of scaffolds with $200 \mathrm{~nm}$ fiber topography. Biomaterials, 2014. 35(9): $\mathrm{p}$. 2837-50.

37. Hotaling, N.A., et al., Nanofiber Scaffold-Based Tissue-Engineered Retinal Pigment Epithelium to Treat Degenerative Eye Diseases. J Ocul Pharmacol Ther, 2016. 32(5): p. 272-85.

38. Sarks, J.P., S.H. Sarks, and M.C. Killingsworth, Evolution of soft drusen in age-related macular degeneration. Eye (Lond), 1994. 8 ( Pt 3): p. 269-83.

39. Curcio, C.A., Soft Drusen in Age-Related Macular Degeneration: Biology and Targeting Via the Oil Spill Strategies. Invest Ophthalmol Vis Sci, 2018. 59(4): p. Amd160-amd181.

40. Starita, C., et al., Localization of the site of major resistance to fluid transport in Bruch's membrane. Invest Ophthalmol Vis Sci, 1997. 38(3): p. 762-7.

41. Rudolf, M., et al., Prevalence and morphology of druse types in the macula and periphery of eyes with age-related maculopathy. Invest Ophthalmol Vis Sci, 2008. 49(3): p. 1200-9.

42. van der Schaft, T.L., et al., Histologic features of the early stages of age-related macular degeneration. A statistical analysis. Ophthalmology, 1992. 99(2): p. 278-86.

43. Mullins, R.F., et al., Drusen associated with aging and age-related macular degeneration contain proteins common to extracellular deposits associated with atherosclerosis, elastosis, amyloidosis, and dense deposit disease. Faseb j, 2000. 14(7): p. 835-46.

44. Rudolf, M., et al., Apolipoprotein A-I Mimetic Peptide L-4F Removes Bruch's Membrane Lipids in Aged Nonhuman Primates. Invest Ophthalmol Vis Sci, 2019. 60(2): p. 461-472.

45. Vavvas, D.G., et al., Regression of Some High-risk Features of Age-related Macular Degeneration (AMD) in Patients Receiving Intensive Statin Treatment. EBioMedicine, 2016. 5: p. 198-203. 
46. Delcourt, C., et al., Associations of cardiovascular disease and its risk factors with age-related macular degeneration: the POLA study. Ophthalmic Epidemiol, 2001. 8(4): p. 237-49.

47. Klein, R., et al., Lipids, lipid genes, and incident age-related macular degeneration: the three continent age-related macular degeneration consortium. Am J Ophthalmol, 2014. 158(3): p. 513-24.e3.

48. Abalain, J.H., et al., Is age-related macular degeneration associated with serum lipoprotein and lipoparticle levels? Clin Chim Acta, 2002. 326(1-2): p. 97-104.

49. Yehoshua, Z., et al., Systemic complement inhibition with eculizumab for geographic atrophy in age-related macular degeneration: the COMPLETE study. Ophthalmology, 2014. 121(3): p. 693-701.

50. Virgili, G., et al., Laser treatment of drusen to prevent progression to advanced age-related macular degeneration. Cochrane Database Syst Rev, 2015(10): p. Cd006537.

51. Garcia Filho, C.A., et al., Change in drusen volume as a novel clinical trial endpoint for the study of complement inhibition in age-related macular degeneration. Ophthalmic Surg Lasers Imaging Retina, 2014. 45(1): p. 18-31.

52. Falavarjani, K.G. and Q.D. Nguyen, Adverse events and complications associated with intravitreal injection of anti-VEGF agents: a review of literature. Eye (Lond), 2013. 27(7): p. 787-94.

53. Huang, X. and Y. Chau, Intravitreal nanoparticles for retinal delivery. Drug Discov Today, 2019. 24(8): p. 1510-1523.

54. Polat, O., et al., Factors Affecting Compliance to Intravitreal Anti-Vascular Endothelial Growth Factor Therapy in Patients with Age-Related Macular Degeneration. Turk J Ophthalmol, 2017. 47(4): p. 205-210.

55. $\mathrm{Yu}, \mathrm{Y}$., et al., Long-term therapeutic effect in nonhuman primate eye from a single injection of anti-VEGF controlled release hydrogel. Bioeng Transl Med, 2019. 4(2): p. e10128.

56. Karumanchi, D.K., et al., Rational design of liposomes for sustained release drug delivery of bevacizumab to treat ocular angiogenesis. Journal of Drug Delivery Science and Technology, 2018. 47: p. 275-282.

57. Zhang, L., et al., Coaxial Electrospray of Ranibizumab-Loaded Microparticles for Sustained Release of Anti-VEGF Therapies. PLOS ONE, 2015. 10(8): p. e0135608.

58. Gupta, P. and K. Yadav, Applications of microneedles in delivering drugs for various ocular diseases. Life Sciences, 2019. 237: p. 116907.

59. Sapino, S., et al., Ocular Drug Delivery: A Special Focus on the Thermosensitive Approach. Nanomaterials (Basel), 2019. 9(6).

60. Balachandra, A., et al., A biocompatible reverse thermoresponsive polymer for ocular drug delivery. Drug Deliv, 2019. 26(1): p. 343-353.

61. Cauldbeck, H., et al., Modulated release from implantable ocular silicone oil tamponade drug reservoirs. J Polym Sci A Polym Chem, 2018. 56(8): p. 938-946.

62. Karim-Zade, K., et al., Retinal Detachment After Removal of Silicone Oil Following Macular Translocation With 360 degree Retinotomy for Neovascular Age-Related Macular Degeneration. Investigative Ophthalmology \& Visual Science, 2005. 46(13): p. 5500-5500.

63. Cunha-Vaz, J., R. Bernardes, and C. Lobo, Blood-retinal barrier. Eur J Ophthalmol, 2011. 21 Suppl 6: p. S3-9.

64. Delie, F., et al., Comparison of two methods of encapsulation of an oligonucleotide into poly(D,L-lactic acid) particles. Int J Pharm, 2001. 214(1-2): p. 25-30.

65. Jo, D.H., T.G. Lee, and J.H. Kim, Nanotechnology and nanotoxicology in retinopathy. Int J Mol Sci, 2011. 12(11): p. 8288-301.

66. Birch, D. and F. Liang, Age-related macular degeneration: A target for nanotechnology derived medicines. International journal of nanomedicine, 2007. 2: p. 65-77.

67. Liu, J., et al., Linear Polyethylenimine-Based DNA Nanoparticle Delivery Into Mouse Retinas. Investigative Ophthalmology \& Visual Science, 2006. 47(13): p. 844-844. 
68. Bourges, J.-L., et al., Ocular Drug Delivery Targeting the Retina and Retinal Pigment Epithelium Using Polylactide Nanoparticles. Investigative Ophthalmology \& Visual Science, 2003. 44(8): p. 3562-3569.

69. Patel, S., et al., Lipid nanoparticles for delivery of messenger RNA to the back of the eye. J Control Release, 2019. 303: p. 91-100.

70. Zhang, L., et al., Pharmacokinetics and tolerance study of intravitreal injection of dexamethasone-loaded nanoparticles in rabbits. Int J Nanomedicine, 2009. 4: p. 175-83.

71. Varshochian, R., et al., Albuminated PLGA nanoparticles containing bevacizumab intended for ocular neovascularization treatment. Journal of Biomedical Materials Research Part A, 2015. 103(10): p. 3148-3156.

72. $\mathrm{Xu}, \mathrm{J} .$, et al., Inhibitory efficacy of intravitreal dexamethasone acetate-loaded PLGA nanoparticles on choroidal neovascularization in a laser-induced rat model. J Ocul Pharmacol Ther, 2007. 23(6): p. 527-40.

73. Tabatabaei, S.N., et al., Co-delivery of miR-181a and melphalan by lipid nanoparticles for treatment of seeded retinoblastoma. J Control Release, 2019. 298: p. 177-185.

74. Axitinib-Loaded Poly(Lactic-Co-Glycolic Acid) Nanoparticles for Age-Related Macular Degeneration: Formulation Development and In Vitro Characterization. ASSAY and Drug Development Technologies, 2019. 17(4): p. 167-177.

75. Paclitaxel Albumin-Stabilized Nanoparticle Formulation in Treating Patients With Metastatic Melanoma of the Eye That Cannot Be Removed By Surgery.

https://ClinicalTrials.gov/show/NCT00738361.

76. Topical Dexamethasone - Cyclodextrin Microparticle Eye Drops for Diabetic Macular Edema. https://ClinicalTrials.gov/show/NCT01523314.

77. Ghosh Chaudhuri, R. and S. Paria, Core/Shell Nanoparticles: Classes, Properties, Synthesis Mechanisms, Characterization, and Applications. Chemical Reviews, 2012. 112(4): p. 23732433.

78. Hayes, R., et al., Core-shell particles: preparation, fundamentals and applications in high performance liquid chromatography. J Chromatogr A, 2014. 1357: p. 36-52.

79. Jegede, O.E., R.F. Cochrane, and A.M. Mullis, Metastable monotectic phase separation in CoCu alloys. Journal of Materials Science, 2018. 53(16): p. 11749-11764.

80. Ideta, R., et al., Nanotechnology-based photodynamic therapy for neovascular disease using a supramolecular nanocarrier loaded with a dendritic photosensitizer. Nano Lett, 2005. 5(12): p. 2426-31.

81. Haneef, A.S. and S. Downes, Controlling Fiber Morphology and Scaffold Design for Treatment of Dry Age-Related Macular Degeneration. International Journal of Polymeric Materials, 2014. 63(18).

82. Haneef, A.S. and S. Downes, Assessing the Suitability of Electrospun Poly(Ethylene Terephthalate) and Polystyrene as Cell Carrier Substrates for Potential Subsequent Implantation as a Synthetic Bruch's Membrane. International Journal of Polymeric Materials, 2015. 64(6).

83. McCormick, R., et al., Optimisation of a Novel Bio-Substrate as a Treatment for Atrophic AgeRelated Macular Degeneration. Frontiers in Bioengineering and Biotechnology, 2020. 8(456). 